\title{
The role of mediator subunit 12 in tumorigenesis and cancer therapeutics (Review)
}

\author{
CRISTIAN G. GONZALEZ ${ }^{*}$, SHIVANI AKULA ${ }^{2 *}$ and MARIEKE BURLESON ${ }^{1}$ \\ Departments of ${ }^{1}$ Biology and ${ }^{2}$ Chemistry, University of The Incarnate Word, San Antonio, TX 78209, USA
}

Received October 21, 2021; Accepted December 14, 2021

DOI: 10.3892/ol.2022.13194

\begin{abstract}
Mediator complex subunit 12 (MED12) is a subunit of Mediator, a large multi-subunit protein complex that acts an important regulator of transcription. Specifically, MED12 is an integral part of the kinase module of Mediator along with MED13, CyclinC (CycC) and CDK8. Structural studies have indicated that MED12 makes a direct connection to $\mathrm{CycC}$ through a specific interface and thereby functions to create a link between MED13 and CycC-CDK8. Disruption of the MED12-CycC interface often leads to dysregulated CDK8 kinase activity, which has important physiological implications. For example, a number of studies have indicated that mutations within MED12 can lead to the formation of benign or malignant tumors, either as a result of MED12-CycC disruption or through distinct independent mechanisms. Furthermore, recent studies have indicated that the $\mathrm{N}$-terminal portion of MED12 forms a direct connection to CDK8. Mutations within MED12 do not appear to disrupt the physical connection to CDK8, but rather abrogate CDK8 kinase activity. Thus, mutations in MED12 can cause disruption of CDK8 kinase activity through two separate mechanisms. The aim of the present review article was to discuss the MED12 mutational landscape in a variety of benign and malignant tumors, as well as the mechanistic basis behind tumorigenesis. Furthermore, the link between MED12 and drug resistance has also been discussed, as well as potential cancer therapeutics related to MED12-altered tumors.
\end{abstract}

\section{Contents}

1. Introduction

2. MED12 mutations and tumorigenesis

Correspondence to: Dr Marieke Burleson, Department of Biology, University of The Incarnate Word, 4301 Broadway, San Antonio, TX 78209, USA

E-mail: mburleso@uiwtx.edu

${ }^{*}$ Contributed equally

Key words: MED12, tumorigenesis, cancer, therapeutics, mediator
3. Therapeutics

4. Conclusions

\section{Introduction}

Med12 is an X-linked gene that provides instructions for making a protein called Mediator complex subunit 12 (MED12). Mediator is a large multi-subunit complex that is critical for gene regulation. MED12 plays an important role in Mediator function by activating and repressing transcription through regulation of RNA polymerase II (Fig. 1A) (1,2). The structure of Mediator includes a dissociable kinase module, comprised of four subunits: MED13, MED12, CyclinC (CycC) and CDK8. Association or dissociation of the kinase module is an important indicator of transcriptional regulation with activation or repression occurring in a context-dependent manner $(3,4)$. The exact reasons behind context-dependent activation vs. repression are currently unclear, but appear to involve induced conformational changes within core Mediator that ultimately affect the ability of Mediator to interact with Polymerase II (5-9). Earlier studies have indicated that MED12 functions as a link between MED13 and CycC-CDK8, with MED12 making a direct connection to CycC $(8,10)$ (Fig. 1A). Furthermore, it has been shown that the exact configuration of the kinase module has important implications for CDK8 kinase activity as CDK8 has been reported to only be fully activated when it is bound to $\mathrm{CycC}$ and MED12 (8,10,11). Specifically, CycC contains a specified surface groove that acts as the docking site for MED12 (8). Mutagenic disruption of the MED12-CycC interface uncouples $\mathrm{CycC}-\mathrm{CDK} 8$ from Mediator, which in turn greatly diminishes CDK8 kinase activity; thus indicating that the MED12-CycC interface plays a critical role in regulating CDK8 kinase activity (8) (Fig. 1B). However, these studies have been complicated as recent findings determined that the N-terminal domain of MED12 directly binds to CDK8 thereby positioning an activation helix close to the T-loop in CDK8 (12,13) (Fig. 1A). Furthermore, these studies reported that MED12 mutations do not disrupt the MED12-CDK8 interaction, but rather alter the positioning of the activation helix thereby diminishing CDK8 kinase activity $(12,13)$ (Fig. 1B). Importantly, in contrast to previous studies, it has been shown that disruption of the MED12-CycC interface has no effect on MED12-dependent CDK8 activation (12). 
Rather, upon MED12 mutation, the entire kinase module becomes destabilized, which ultimately leads to diminished CDK8 kinase activity (12). Nonetheless, all of these studies demonstrate that mutations within MED12 cause conformational changes in the Mediator kinase module that ultimately disrupt CDK8 kinase activity. Consequently, altered CDK8 kinase activity, due to site-specific mutations within MED12, has important implications in gene regulation $(3,4,14)$. Importantly, a number of the genes that show altered expression upon MED12 mutations are associated with several different types of human cancers, thus showing a link between MED12 and tumor formation (15). Therefore, the present review aimed to discuss tumorigenesis as a result of somatic mutations in MED12. Furthermore, the review also discussed drug resistance mechanisms and potential treatment options for MED12-related disorders.

\section{MED12 mutations and tumorigenesis}

Uterine leiomyomas. Uterine leiomyomas, also commonly known as fibroids, are a classification of muscle tumors that are benign. Extensive research has pointed out that most uterine leiomyoma-associated MED12 mutations are located within exon 2, with only some exceptions occurring within exon 1 (15-35) (Table I). Based on the current literature there are two plausible mechanisms behind MED12 mutant-uterine leiomyoma tumorigenesis. First, it is postulated that this mutation disrupts the interaction between MED12 and CyclinC-CDK8 in the kinase module of Mediator (36) (Fig. 1B). Extensive studies have shown that complete CDK8 activation depends on the interaction between $\mathrm{CycC}$ and MED12, with this interaction specifically occurring within the first two exons of MED12 (8,22,24,37-39). Therefore, mutations occurring within MED12 exon 1 or exon 2 often lead to disruption of the interaction between $\mathrm{CycC}$ and MED12, which subsequently leads to reduced CDK8 kinase activity (Fig. 1B). Secondly, recent studies have now postulated that MED12 mutations found in patients with uterine leiomyoma do not affect the MED12-CycC interaction, but rather abolish CDK8 kinase activity through displacement of a MED12-mediated activation loop on CDK8 $(12,13)$ (Fig. 1B). Nonetheless, one common theme among these studies is that reduced CDK8 kinase activity, as a result of MED12 mutations, causes cell cycle dysregulation thereby resulting in abnormal cell growth and ultimately tumor formation (40-42). Further studies will need to be completed to gain a full understanding of the mechanistic basis behind tumorigenesis in uterine leiomyomas as a result of MED12 mutations.

Phyllodes tumors. Phyllodes tumors are fibroepithelial tumors found in the breast, which can include benign, borderline and malignant tumors with $\sim 1$ in every 4 tumors being malignant. MED12 mutations have been identified in up to $80 \%$ of phyllodes tumors with little variance of frequency amongst malignant (77\%), benign (83\%) and borderline $(80 \%)$ tumors $(15,39)$ (Table I). These findings indicate that MED12 mutations play an important role in the development of phyllodes tumors, but do not appear to be involved in the progression from benign grade to malignant grade tumors.
Of note, in another study, patients with MED12-mutant phyllodes tumors showed improved disease-free survival when compared with patients with phyllodes tumors lacking MED12 mutations (43). The reason for this is still unknown, but it may provide insight into the role of MED12 mutations in phyllodes tumor formation and progression. The majority of MED12 mutations found in phyllodes tumors reside within exon 2, which would lead to impairment of CDK8 kinase activity as seen in uterine leiomyomas. However, further studies are required to investigate the mechanistic basis behind MED12 mutant phyllodes tumorigenesis.

Fibroadenomas. Fibroadenomas are a benign breast fibroepithelial tumor commonly found in women $<30$ years old, which occur more frequently than phyllodes tumors. The MED12 mutation patterns of both phyllodes tumors and fibroadenomas are very similar, with missense and deletion-insertion mutations occurring mainly in exon 2 (and a small number in exon 1), with codon 44 being the most commonly mutated $(86 \%)(15,38,44)$ (Table I). Codons 36 and 43 are also affected, but at a much less frequent rate (16). Similar to uterine leiomyomas and phyllodes tumors, exon 2 mutations disrupt CDK8 kinase activity (44). In addition to MED12 mutations, MED12 suppression in fibroadenomas, as shown by RNA interference, enhances the activation of transforming growth factor-b (TGF-b) signaling, which causes activation of mitogen-activated protein kinase/extracellular signal-regulated kinase, ultimately causing resistance to cancer drugs that target this specific pathway (45). These observations thus provide insight into drug resistance mechanisms involving tumors containing MED12 alterations.

Chronic lymphatic leukemia. Chronic lymphatic leukemia (CLL) is a hematological disease that results from the accumulation of mature B lymphocytes in peripheral blood, bone marrow and lymph nodes. The extent of this disease observed in patients varies from being highly indolent, which may never require therapy, to very aggressive and requiring extensive treatment (46). It has been found that $\sim 5-9 \%$ of the mutations found in patients with lymphatic leukemia correspond to mutations in the N-terminus of MED12, specifically within exon 1 and exon 2 (37,46-48) (Table I). CLL-associated MED12 mutations have been found to affect NOTCH signaling through disruption of the MED12-CycC interface (46). NOTCH signaling can act in an oncogenic or tumor suppressive manner depending on cellular context (49-52). CLL-associated MED12 mutations have been shown to increase NOTCH as a direct result of MED12-CycC dissociation and subsequent loss of CDK8 kinase activity $(8,17,37,53)$. Consequently, enhanced NOTCH signaling in these patients increases apoptotic resistance to ultimately promote tumor formation $(46,53)$.

Prostate cancer. Prostate cancer is a hormone-driven cancer regulated through interactions of circulating androgens to the androgen receptor (54). Of note, a link has been established between MED12 overexpression and TGF- $\beta$ signaling in castration-resistant prostate cancer (CRPC), a highly advanced and aggressive type of prostate cancer that is unresponsive to hormone treatment (55). Specifically, 


\section{A}

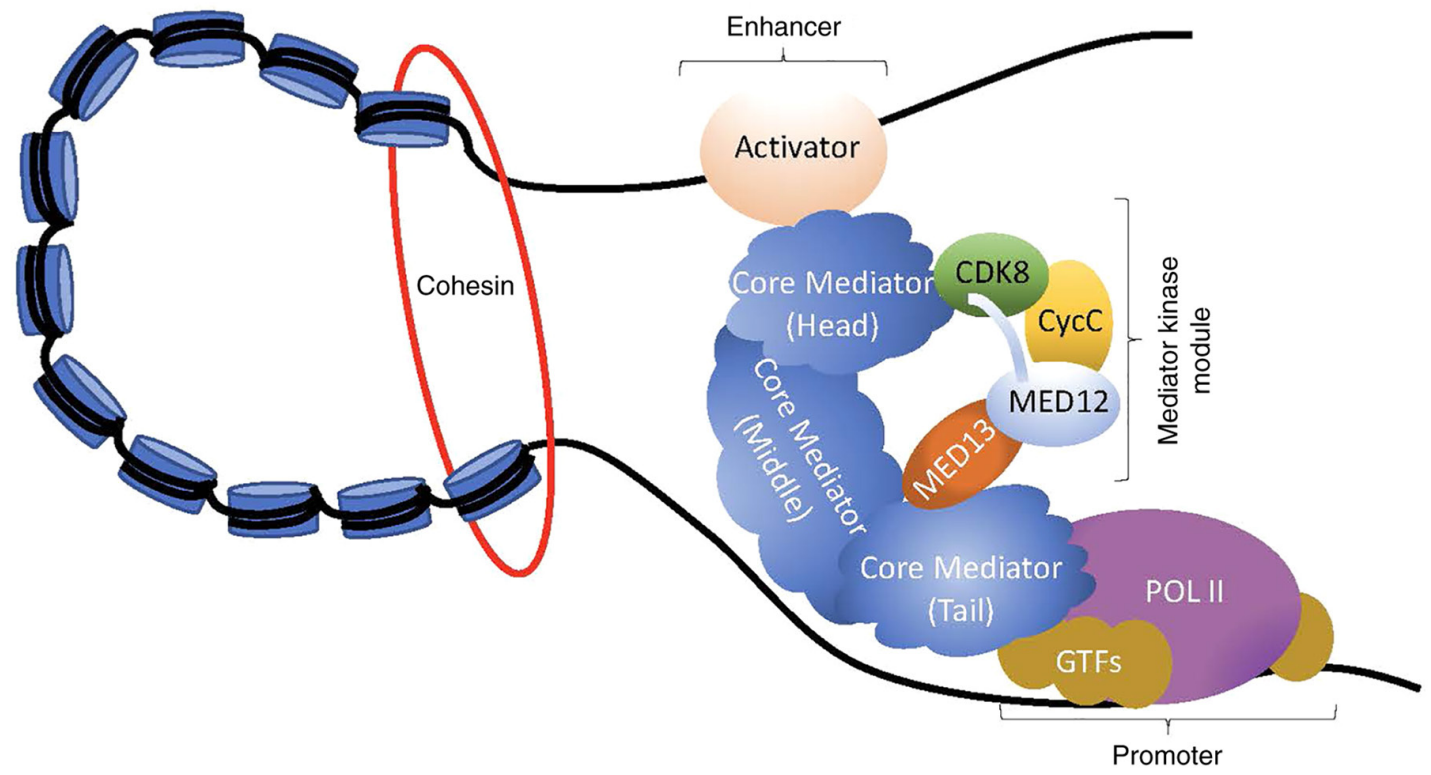

$\mathrm{B}$
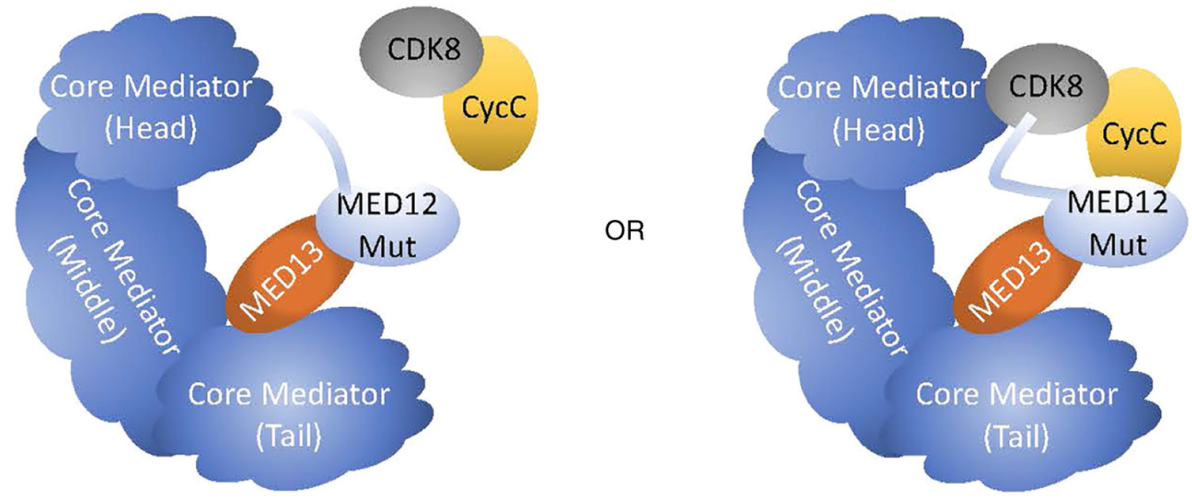

Figure 1. Structure of core Mediator and the kinase module. (A) Mediator is composed of core mediator (head region, middle region, and tail region), and the kinase module. The tail region of core Mediator interacts with POL II and the head region interacts with activator proteins to regulate transcription. The kinase module is made up of CDK8, CycC, MED12, and MED13. (B) Mutations within MED12 (MED12 Mut) disrupt the MED12-CycC interface thereby leading to dissociation of the CycC-CDK8 complex and subsequent inactivation of CDK8 kinase activity (grey CDK8). Alternatively, mutations within MED12 do not disrupt association of MED12 to CycC, but rather promote dissociation of the activation loop to lead to inactivation of CDK8 kinase activity. MED12, Mediator complex subunit 12; POL II, RNA polymerase II; GTFs, general transcription factors; CycC, Cyclin C.

MED12 overexpression, as assayed by immunohistochemistry on tissue microarrays, was observed in 70 distance metastatic and 90 local recurrent CRPC samples (55). MED12 overexpression in these samples was significantly correlated to activated TGF- $\beta$ signaling (55). In addition to MED12 overexpression, MED12 is also mutated in $~ 5 \%$ of prostate cancers (56-58) (Table I). Unlike the previously discussed tumors, MED12 mutations in prostate cancer do not reside within the $\mathrm{N}$-terminus, with the most common mutation found in exon 26 (57). Further studies will need to be performed to determine the mechanistic basis behind prostate cancer-associated MED12 mutations. Of note, however, most prostate cancer-associated MED12 mutations are in close proximity to MED12 mutations found in the $\mathrm{X}$-linked intellectual disability (XLID) disorders, FG, Lujan and Ohdo syndromes (33,59-61). XLID-associated MED12 mutations have previously been shown to impair recruitment of CycC-CDK8 into Mediator, which ultimately promotes hyperactivated Sonic Hedgehog signaling (62-64). It is thus plausible that MED12 mutations in prostate cancer may promote tumorigenesis through a similar mechanism.
Breast and ovarian cancer. Breast and ovarian cancer are two additional examples of cancer subtypes that are primarily driven by hormones; with breast cancer being driven by estrogen and progesterone, and ovarian cancer by estrogen. Recently, MED12 mutations have been found in breast (up to $33.3 \%)$ and ovarian $(\sim 3 \%)$ cancer, however insight into the mechanistic basis behind these mutations is still lacking (65-68) (Table I). One noteworthy finding in ovarian cancer is that MED12 mutations correlate with high tumor mutational burden (TMB-H) as mutations were exclusively found in tumors classified as TMB-H (68). Of note, TMB-H has been linked to the generation of neoantigens and clinical response to immunotherapies in various cancer subtypes (68). Thus, MED12 mutations could serve as a potential biomarker for immunotherapy in ovarian cancer. In breast cancer, MED12 expression levels have been correlated to the regulation of angiotensin converting enzyme 2 (ACE2) levels and subsequent severe acute respiratory syndrome-related coronavirus 2 (SARS-CoV-2) infection (69). The details of this study will be further discussed in the therapeutics section of the current review. 
Table I. Summary of MED12 mutations.

\begin{tabular}{lcll}
\hline Type of tumorigenesis & Prevalence of mutation (\%) & Affected exon(s) & Mode of mechanism \\
\hline Uterine leiomyomas & $37-86$ & Exon 1, Exon 2 & Loss of CDK8 kinase activity \\
Phyllodes tumors & $43-80$ & Exon 2 & Loss of CDK8 kinase activity \\
Fibroadenomas & $59-62$ & Exon 1, Exon 2 & Loss of CDK8 kinase activity \\
Chronic lymphatic leukemia & $5-9$ & Exon 1, Exon 2 & Increased Notch signaling \\
Prostate cancer & $2-5$ & Exon 26 & Possible hyperactivated Sonic Hedgehog \\
& & & signaling? \\
Breast cancer & $3-33$ & Multiple & ?Unknown \\
Ovarian cancer & 50 & Exon 14 & Unknown \\
\hline
\end{tabular}

MED12, Mediator complex subunit 12.

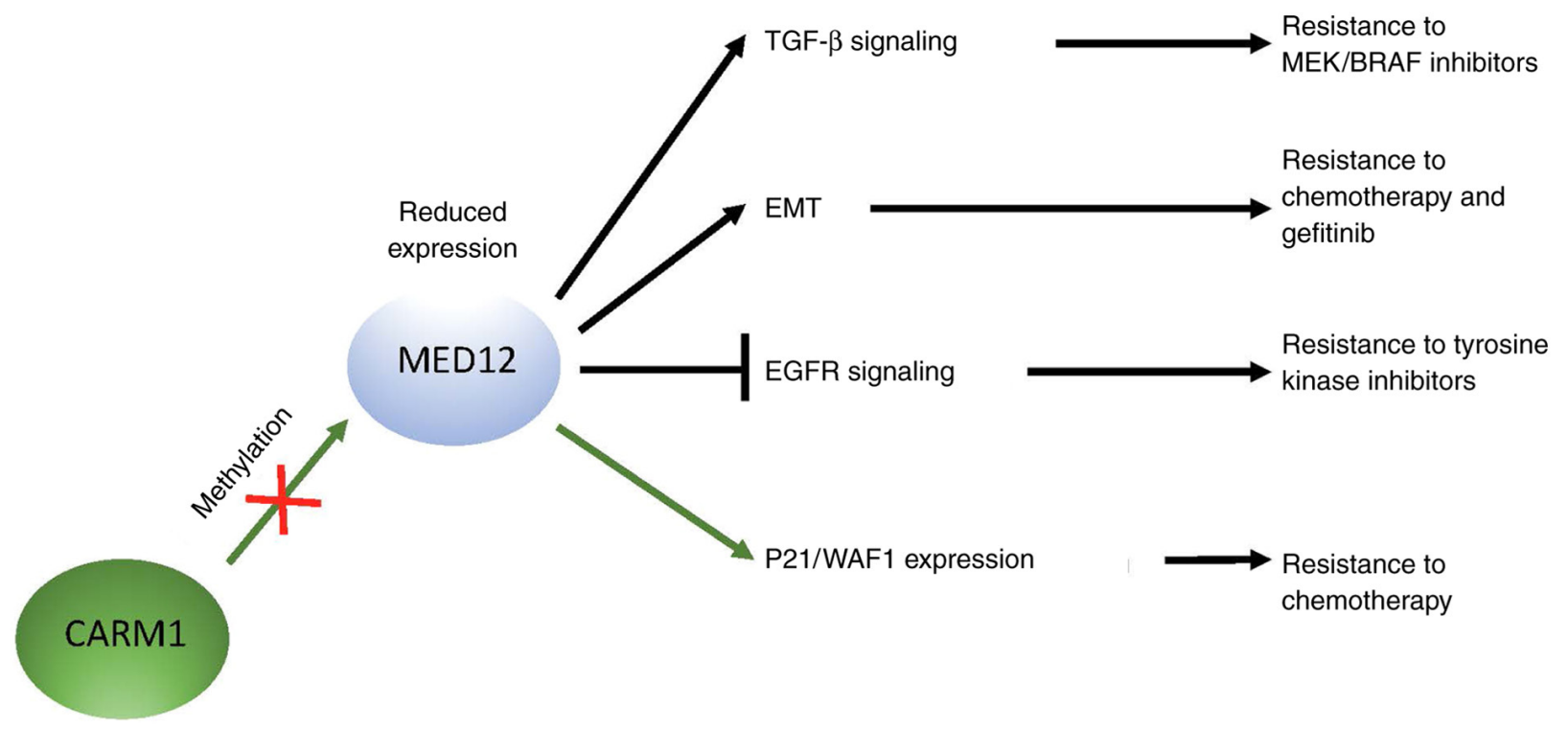

Figure 2. Overview of the role of MED12 in drug resistance. Reduced MED12 expression can lead to upregulation of TGF- $\beta$ signaling or promote the EMT which subsequently leads to resistance to MEK/BRAF inhibitors or chemotherapy/gefitinib, respectively. Reduced MED12 expression also inhibits EGFR signaling leading to resistance to tyrosine kinase inhibitors. Inhibition of CARM1-mediated MED12 methylation promotes downregulation of MED12 and a subsequent upregulation of P21/WAF1 expression to drive chemotherapy resistance. MED12, Mediator complex subunit 12; EMT, epithelial to mesenchymal transition; EGFR, epidermal growth factor receptor; TGF- $\beta$, transforming growth factor- $\beta$; CARM1, coactivator-associated arginine methyltransferase 1.

\section{Therapeutics}

Drug resistance. Several studies have found that loss of genomic and non-genomic functions of MED12 are associated with the development of resistance to chemotherapeutics. A specific example of this is seen in non-small cell lung cancer, where loss of MED12, as assayed by RNA interference, causes drug resistance, by increasing the level of TGF- $\beta$ receptor II (R2) (45,70). MED12 physically interacts with TGF- $\beta$ R2 in the cytoplasm, which results in a reduction of TGF- $\beta$ signaling (45). Consequently, reduced MED12 expression promotes TGF- $\beta$ signaling that has been linked directly to drug resistance, including resistance to $\mathrm{MEK}$ and $\mathrm{BRAF}$ inhibitors, downstream effectors in the TGF- $\beta$ signaling pathway (45) (Fig. 2). Furthermore, reduced MED12 expression is also associated with an epithelial-to-mesenchymal-like phenotype that has been correlated to chemotherapy resistance in colon cancer and gefitinib resistance in lung cancer (45) (Fig. 2).
Since MED12 mutations abrogate CDK8 kinase activity, either due to a disruption of MED12-CycC or a displacement of the activation loop of CDK8, it can be argued that loss of MED12 expression mimics a MED12 mutant setting. Thus, these results indicate that inhibition of TGF- $\beta$ signaling in cancers with either reduced MED12 expression or MED12 mutations could prove to be a beneficial therapeutic strategy.

In another study, MED12 overexpression was correlated to improved disease prognosis in human breast cancer (71). Specifically, this was shown to involve the interaction of MED12 with coactivator-associated arginine methyltransferase 1 (CARM 1) for which MED12 is known to be a substrate (71). CARM1 and MED12 expression show a positive correlation, and high expression of both predicts improved prognosis in patients with breast cancer after chemotherapy (71). CARM1 methylates MED12 at residues R1862 and R1912, and mutations within these sites promote MED12 downregulation, which results in resistance to chemotherapy drugs $(71,72)$ 
(Fig. 2). The drug response mechanism shown in these cells is distinct from the mechanism involving TGF- $\beta$ signaling activation as methylated MED12 represses p21/WAF1 transcription, which ultimately promotes cell cycle progression and cell proliferation (71). Overall, this study showed that MED12 expression levels, as a result of methylation status, may serve as a potential biomarker to determine severity of disease and potential drug resistance.

Finally, in a recent study, a genome-wide clustered regularly interspaced short palindromic repeats (CRISPR) knockout screen was employed in HeLa and MCF10A (non-malignant breast epithelial) cells to identify mechanisms that regulate resistance to ataxia telangiectasia and $\operatorname{Rad} 3$ related inhibitors (ATRi) (73). ATR plays a pivotal role in the DNA damage response pathway thereby making it an attractive target for chemotherapeutics. For example, ATRi have been shown to be highly efficient in targeting cancer cells that have a large amount of DNA damage and are deficient in ATM/p53 (74-76). Through the CRISPR knockout screen, MED12 was identified as playing a crucial role in ATRi resistance (73). Specifically, MED12 knockout or knockdown was shown to increase the CldU-to-IdU ratio, thus indicating that loss of MED12 promotes restoration of replication fork speed in the presence of ATRi (73). Overall, this study provides further insight into the pivotal role of MED12 in cancer drug resistance pathways.

MED12 and SARS-CoV-2 infection. Patients with cancer have been shown to be at an increased risk for severe symptoms from SARS-CoV-2 infection, although the precise reasons behind this are unclear (69). Of note, a recent study has found that chemotherapy treatment gradually decreases MED12 levels in breast cancer cells (CAL-51 cells), which subsequently promotes an increase in ACE2 expression (69). Since ACE2 plays a critical role in viral entry of SARS-CoV-2, this finding demonstrates a link between chemotherapy treatment, MED12 expression and the likelihood of viral infection. Furthermore, upon further investigation, it was found that CRISPR-mediated knockout of MED12 affects the expression of 31 different viral-dependent host factors, including centromere protein $\mathrm{F}$ and numerous fibroblast growth factors (69). Thus, MED12 plays a role in blocking SARS-CoV-2 infection through the repression of ACE2 expression to control viral entry and by potentiating broad anti-viral defense pathways in the host.

Epidermal growth factor receptor (EGFR) signaling. EGFR signaling regulates growth, proliferation, survival and differentiation in cells. This transmembrane tyrosine kinase receptor is commonly upregulated or mutated in various cancers, resulting in the activation of several oncogenic pathways. Of note, MED12 can bind to the EGFR promoter through Mediator-polymerase II complex to stimulate EGFR transcription and thus subsequently promote tumor proliferation (77). MED12 and EGFR expression levels are directly related to MED12 knockdown, causing decreased EGFR signaling, which in turn causes tumor dormancy (77). Specifically, in ovarian cancer cells, depletion of MED12 was enough to cause tumor dormancy by causing the cell cycle to arrest in the $G_{0}-G_{1}$ phase (77). This finding is of importance in cancer therapeutics as several studies have suggested that tumor relapse may be prevented by inhibiting the conversion of tumor dormancy to tumor proliferation $(77,78)$. Thus, it may be beneficial to keep tumor cells in the dormant state and prevent the relapse of cancer through the use of MED12 inhibitors. However, further studies must be conducted to confirm this strategy as a therapeutic option.

Genetic testing. DNA sequencing is key in modern cancer therapeutics as it allows for the generation of personalized treatment options. In one study, researchers investigated the efficacy of genetic sequencing on patients with non-small cell lung cancer who developed resistance to EGFR tyrosine kinase inhibitors (Fig. 2) (79). Of note, a direct correlation was found between reduced MED12 expression and drug resistance in these patients (79). The researchers found that deep sequencing analysis of tumors was efficient in identifying drug response and resistance biomarkers as well as clonal and subclonal genetic populations (79). The identification of biomarkers allows for serial biomarker analysis of samples gathered from each patient to guide the initial and subsequent treatments to prevent or overcome drug resistance. It is important that this genotyping is enriched with additional biomarkers to predict signaling pathways that crosstalk and are involved in adaptive resistance to treatment. Targeting these signaling pathways, and adapting the treatment based on serial genetic analysis, might be a more effective alternative to EGFR tyrosine kinase inhibitor monotherapy (79). This study thus provides important insight into the efficacy and use of genotyping in cancer therapeutics as well as the role of MED12 in potential drug resistance.

\section{Conclusions}

MED12 functions in regulating cell growth signals, early development and differentiation. As a result of these important physiological functions, mutations within MED12 are commonly seen in several different cancers and benign tumors, including uterine leiomyomas, phyllodes tumors, breast fibroadenomas, lymphatic leukemia, prostate, breast and ovarian cancer. One theme that is common to most of these tumors, with the exception of prostate cancer, is that the mutations occur within exon 1 or exon 2, which leads to a reduction of CDK8 kinase activity; though it is currently unclear if this reduction is due to disruption of MED12-CycC or a displacement of the activation loop on CDK8 (12,13,16-32,34,35,39,44,46-48). The resulting mechanistic basis behind tumorigenesis as a result of loss of CDK8 kinase activity varies between the different tumor subtypes. At least one study has shown that loss of CDK8 kinase activity leads to dysregulated NOTCH signaling to promote lymphatic leukemia (46). It is of importance to note that other studies have investigated the relationship between CDK8 kinase activity and tumorigenesis in a MED12-independent context in a variety of different cancers $(80,81)$. Nonetheless, further studies are required to study the mechanisms behind MED12-mediated loss of CDK8 kinase activity and subsequent tumorigenesis.

One theme that is noteworthy is the fact that the exon 1/exon 2 MED12 mutations are restricted primarily, with the exception of lymphatic leukemia, to hormone-driven tumors with mesenchymal stem cell origins. The reasons underlying this finding are currently unclear, but they 
do suggest that MED12 plays an important role in the development and/or differentiation of this progenitor lineage. Of note, the only tumor subtype that has provided clues as to the mechanistic basis behind exon1/exon 2 MED12-mutation driven tumorigenesis stems from studies in lymphatic leukemia, the only non-hormone-driven tumor. Future studies are warranted to determine whether the mechanism underlying hormone-driven tumorigenesis is similar or distinct to that observed in lymphatic leukemia. Furthermore, the relationship between exon1/exon 2 mutations and the exon 26 mutations found in prostate cancer is currently unclear. Prostate cancer and exon1/exon2-mutant tumors are both hormone-driven, so it is unclear as to why mutations in these tumors are found in very distinct regions of MED12.

Although information regarding the mechanistic basis behind MED12-mutant tumorigenesis is still lacking, there are several studies that have begun to investigate the role of MED12 in potential cancer therapeutics. One important finding is that reduced MED12 expression can lead to drug resistance, including resistance to MEK, BRAF and tyrosine kinase inhibitors; thereby, providing important insight into drug treatment for patients who display reduced MED12 expression (45,79). Congruently, potential has been demonstrated in the importance of genetic testing, specifically in determining the mutant or expression landscape of MED12. Not only will this provide clues as to potential drug resistance mechanisms, but it will also provide insight into potential treatment strategies. Ultimately, future studies will need to be performed to determine whether MED12 itself, the MED-CycC interface or the MED12-CDK8 connection can serve as potential therapeutic targets for cancer treatment.

\section{Acknowledgements}

Not applicable.

\section{Funding}

No funding was received.

\section{Availability of data and materials}

Not applicable.

\section{Author contributions}

SA and CGG wrote the manuscript. MB provided guidance and performed all editing and formatting. Data authentication is not applicable. All authors read and approved the final manuscript.

\section{Ethics approval and consent to participate}

Not applicable.

\section{Patient consent for publication}

Not applicable.

\section{Competing interests}

The authors declare that they have no competing interests.

\section{References}

1. Allen BL and Taatjes DJ: The mediator complex: A central integrator of transcription. Nat Rev Mol Cell Biol 16: 155-166, 2015.

2. Kornberg RD: Mediator and the mechanism of transcriptional activation. Trends Biochem Sci 30: 235-239, 2005.

3. Mo X, Kowenz-Leutz E, Xu H and Leutz A: Ras induces mediator complex exchange on C/EBP beta. Mol Cell 13: 241-250, 2004.

4. Pavri R, Lewis B, Kim TK, Dilworth FJ, Erdjument-Bromage H, Tempst P, de Murcia G, Evans R, Chambon P and Reinberg D: PARP-1 determines specificity in a retinoid signaling pathway via direct modulation of mediator. Mol Cell 18: 83-96, 2005.

5. Bernecky C, Grob P, Ebmeier CC, Nogales E and Taatjes DJ: Molecular architecture of the human Mediator-RNA polymerase II-TFIIF assembly. PLoS Biol 9: e1000603, 2011.

6. Elmlund H, Baraznenok V, Lindahl M, Samuelsen CO, Koeck PJ, Holmberg S, Hebert $\mathrm{H}$ and Gustafsson CM: The cyclin-dependent kinase 8 module sterically blocks Mediator interactions with RNA polymerase II. Proc Natl Acad Sci USA 103: 15788-15793, 2006.

7. Knuesel MT, Meyer KD, Bernecky C and Taatjes DJ: The human CDK8 subcomplex is a molecular switch that controls Mediator coactivator function. Genes Dev 23: 439-451, 2009.

8. Turunen M, Spaeth JM, Keskitalo S, Park MJ, Kivioja T, Clark AD, Mäkinen N, Gao F, Palin K, Nurkkala H, et al: Uterine leiomyoma-linked MED12 mutations disrupt mediator-associated CDK activity. Cell Rep 7: 654-660, 2014.

9. Wang X, Sun Q, Ding Z, Ji J, Wang J, Kong X, Yang J and Cai G: Redefining the modular organization of the core Mediator complex. Cell Res 24: 796-808, 2014.

10. Knuesel MT, Meyer KD, Donner AJ, Espinosa JM and Taatjes DJ: The human CDK8 subcomplex is a histone kinase that requires Med12 for activity and can function independently of mediator. Mol Cell Biol 29: 650-661, 2009.

11. Xu W and Ji JY: Dysregulation of CDK8 and Cyclin C in tumorigenesis. J Genetics 38: 439-452, 2011.

12. Klatt F, Leitner A, Kim IV, Ho-Xuan H, Schneider EV, Langhammer F, Weinmann R, Müller MR, Huber R, Meister G and Kuhn CD: A precisely positioned MED12 activation helix stimulates CDK8 kinase activity. Proc Natl Acad Sci USA 117: 2894-2905, 2020.

13. Li YC, Chao TC, Kim HJ, Cholko T, Chen SF, Li G, Snyder L, Nakanishi K, Chang CE, Murakami K, et al: Structure and noncanonical Cdk8 activation mechanism within an Argonaute-containing Mediator kinase module. Sci Adv 7: eabd4484, 2021.

14. Holstege FC, Jennings EG, Wyrick JJ, Lee TI, Hengartner CJ, Green MR, Golub TR, Lander ES and Young RA: Dissecting the regulatory circuitry of a eukaryotic genome. Cell 95: 717-728, 1998.

15. Clark AD, Oldenbroek $M$ and Boyer TG: Mediator kinase module and human tumorigenesis. Crit Rev Biochem Mol Biol 50: 393-426, 2015.

16. Ajabnoor GMA, Mohammed NA, Banaganapalli B, Abdullah LS, Bondagji ON, Mansouri N, Sahly NN, Vaidyanathan V, Bondagji N, Elango R and Shaik NA: Expanded somatic mutation spectrum of MED12 gene in uterine leiomyomas of Saudi Arabian Women. Front Genet 9: 552, 2018.

17. Banaganapalli B, Mohammed K, Khan IA, Al-Aama JY, Elango R and Shaik NA: A Computational protein phenotype prediction approach to analyze the deleterious mutations of human MED12 gene. J Cell Biochem 117: 2023-2035, 2016.

18. Croce S and Chibon F: MED12 and uterine smooth muscle oncogenesis: State of the art and perspectives. Eur J Cancer 51: 1603-1610, 2015.

19. de Graaff MA, Cleton-Jansen AM, Szuhai K and Bovée JV: Mediator complex subunit 12 exon 2 mutation analysis in different subtypes of smooth muscle tumors confirms genetic heterogeneity. Hum Pathol 44: 1597-1604, 2013.

20. Heinonen HR, Sarvilinna NS, Sjöberg J, Kämpjärvi K, Pitkänen E, Vahteristo P, Mäkinen N and Aaltonen LA: MED12 mutation frequency in unselected sporadic uterine leiomyomas. Fertil Steril 102: 1137-1142, 2014. 
21. Je EM, Kim MR, Min KO, Yoo NJ and Lee SH: Mutational analysis of MED12 exon 2 in uterine leiomyoma and other common tumors. Int J Cancer 131: E1044-E1047, 2012.

22. Kämpjärvi K, Mäkinen N, Kilpivaara O, Arola J, Heinonen HR, Böhm J, Abdel-Wahab O, Lehtonen HJ, Pelttari LM, Mehine M, et al: Somatic MED12 mutations in uterine leiomyosarcoma and colorectal cancer. Br J Cancer 107: 1761-1765, 2012

23. Li N, Fassl A, Chick J, Inuzuka H, Li X, Mansour MR, Liu L, Wang H, King B, Shaik S, et al: Cyclin C is a haploinsufficient tumour suppressor. Nat Cell Biol 16: 1080-1091, 2014

24. Mäkinen N, Heinonen HR, Moore S, Tomlinson IP, van der Spuy ZM and Aaltonen LA: MED12 exon 2 mutations are common in uterine leiomyomas from South African patients. Oncotarget 2: 966-969, 2011.

25. Mäkinen N, Mehine M, Tolvanen J, Kaasinen E, Li Y,Lehtonen HJ, Gentile M, Yan J, Enge M, Taipale M, et al: MED12, the mediator complex subunit 12 gene, is mutated at high frequency in uterine leiomyomas. Science 334: 252-255, 2011.

26. Mäkinen N, Vahteristo P, Kämpjärvi K, Arola J, Bützow R and Aaltonen LA: MED12 exon 2 mutations in histopathological uterine leiomyoma variants. Eur J Hum Genet 21: 1300-1303, 2013.

27. Matsubara A, Sekine S, Yoshida M, Yoshida A, Taniguchi H Kushima R, Tsuda $\mathrm{H}$ and Kanai Y: Prevalence of MED12 mutations in uterine and extrauterine smooth muscle tumours. Histopathology 62: 657-661, 2013.

28. McGuire MM, Yatsenko A, Hoffner L, Jones M, Surti U and Rajkovic A: Whole exome sequencing in a Random Sample of North American Women with leiomyomas identifies MED12 mutations in majority of uterine leiomyomas. PLoS One 7: e33251, 2012.

29. Mehine M, Kaasinen E, Mäkinen N, Katainen R, Kämpjärvi K, Pitkänen E, Heinonen HR, Bützow R, Kilpivaara O, Kuosmanen A, et al: Characterization of uterine leiomyomas by whole-genome sequencing. N Engl J Med 369: 43-53, 2013.

30. Pérot G, Croce S, Ribeiro A, Lagarde P, Velasco V, Neuville A, Coindre JM, Stoeckle E, Floquet A, MacGrogan G and Chibon F: MED12 alterations in both human benign and malignant uterine soft tissue tumors. PLoS One 7: e40015, 2012.

31. Ravegnini G, Mariño-Enriquez A, Slater J, Eilers G, Wang Y, Zhu M, Nucci MR, George S, Angelini S, Raut CP and Fletcher JA: MED12 mutations in leiomyosarcoma and extrauterine leiomyoma. Mod Pathol 26: 743-749, 2013.

32. Schwetye KE, Pfeifer JD and Duncavage EJ: MED12 exon 2 mutations in uterine and extrauterine smooth muscle tumors. Hum Pathol 45: 65-70, 2014.

33. Wang H, Shen Q, Ye LH and Ye J: MED12 mutations in human diseases. Protein Cell 4: 643-646, 2013.

34. Wang L, Hu S, Xin F, Zhao H, Li G, Ran W, Xing X and Wang J: MED12 exon 2 mutation is uncommon in intravenous leiomyomatosis: Clinicopathologic features and molecular study. Hum Pathol 99: 36-42, 2020

35. Zhang Q, Ubago J, Li L, Guo H, Liu Y, Qiang W, Kim JJ, Kong B and Wei JJ: Molecular analyses of 6 different types of uterine smooth muscle tumors: Emphasis in atypical leiomyoma. Cancer 120: 3165-3177, 2014

36. Park MJ, Shen H, Spaeth JM, Tolvanen JH, Failor C, Knudtson JF, McLaughlin J, Halder SK, Yang Q, Bulun SE, et al: Oncogenic exon 2 mutations in Mediator subunit MED12 disrupt allosteric activation of cyclin C-CDK8/19. J Biol Chem 293: 4870-4882, 2018.

37. Kämpjärvi K, Järvinen TM, Heikkinen T, Ruppert AS, Senter L, Hoag KW, Dufva O, Kontro M, Rassenti L, Hertlein E, et al: Somatic MED12 mutations are associated with poor prognosis markers in chronic lymphocytic leukemia. Oncotarget 6 : $1884-1888,2015$

38. Lim WK, Ong CK, Tan J, Thike AA, Ng CC, Rajasegaran V, Myint SS, Nagarajan S, Nasir ND, McPherson JR, et al: Exome sequencing identifies highly recurrent MED12 somatic mutations in breast fibroadenoma. Nat Genet 46: 877-880, 2014.

39. Yoshida M, Sekine S, Ogawa R, Yoshida H, Maeshima A, Kanai Y, Kinoshita T and Ochiai A: Frequent MED12 mutations in phyllodes tumours of the breast. Br J Cancer 112: 1703-1708, 2015.

40. Kishi T, Ikeda A, Koyama N, Fukada J and Nagao R: A refined two-hybrid system reveals that $\mathrm{SCF}(\mathrm{Cdc} 4)$-dependent degradation of Swi5 contributes to the regulatory mechanism of S-phase entry. Proc Natl Acad Sci USA 105: 14497-14502, 2008

41. Porter DC, Farmaki E, Altilia S, Schools GP, West DK, Chen M, Chang BD, Puzyrev AT, Lim CU, Rokow-Kittell R, et al Cyclin-dependent kinase 8 mediates chemotherapy-induced tumor-promoting paracrine activities. Proc Natl Acad Sci USA 109: 13799-13804, 2012.
42. Xu D, Li CF, Zhang X, Gong Z, Chan CH, Lee SW, Jin G, Rezaeian AH, Han F, Wang J, et al: Skp2-macroH2A1-CDK8 axis orchestrates $\mathrm{G} 2 / \mathrm{M}$ transition and tumorigenesis. Nat Commun 6: 6641, 2015

43. Ng CC, Tan J, Ong CK, Lim WK, Rajasegaran V, Nasir ND, Lim JC, Thike AA, Salahuddin SA, Iqbal J, et al: MED12 is frequently mutated in breast phyllodes tumours: A study of 112 cases. J Clin Pathol 68: 685-691, 2015.

44. Darooei M, Khan F, Rehan M, Zubeda S, Jeyashanker E, Annapurna S, Shah A, Maddali S and Hasan Q: MED12 somatic mutations encompassing exon 2 associated with benign breast fibroadenomas and not breast carcinoma in Indian women. J Cell Biochem 120: 182-191, 2019.

45. Huang S, Hölzel M, Knijnenburg T, Schlicker A, Roepman P, McDermott U, Garnett M, Grernrum W, Sun C, Prahallad A, et al: MED12 controls the response to multiple cancer drugs through regulation of TGF- $\beta$ receptor signaling. Cell 151: 937-950, 2012.

46. Wu B, Słabicki M, Sellner L, Dietrich S, Liu X, Jethwa A, Hüllein J, Walther T, Wagner L, Huang Z, et al: MED12 mutations and NOTCH signalling in chronic lymphocytic leukaemia. Br J Haematol 179: 421-429, 2017.

47. Bullerdiek $\mathrm{J}$ and Rommel B: Factors targeting MED12 to drive tumorigenesis? F1000Res 7: 359, 2018.

48. Guièze R, Robbe $\mathrm{P}$, Clifford R, de Guibert S, Pereira B, Timbs A, Dilhuydy MS, Cabes M, Ysebaert L, Burns A, et al: Presence of multiple recurrent mutations confers poor trial outcome of relapsed/refractory CLL. Blood 126: 2110-2117, 2015.

49. Aster JC, Pear WS and Blacklow SC: The varied roles of notch in cancer. Annu Rev Pathol 12: 245-275, 2017.

50. Meurette $O$ and Mehlen P: Notch signaling in the tumor microenvironment. Cancer Cell 34: 536-548, 2018.

51. Nowell CS and Radtke F: Notch as a tumour suppressor. Nat Rev Cancer 17: 145-159, 2017

52. Ntziachristos P, Lim JS, Sage J and Aifantis I: From fly wings to targeted cancer therapies: A centennial for notch signaling. Cancer Cell 25: 318-334, 2014.

53. Rosati E, Sabatini R, Rampino G, Tabilio A, Di Ianni M, Fettucciari K, Bartoli A, Coaccioli S, Screpanti I and Marconi P: Constitutively activated Notch signaling is involved in survival and apoptosis resistance of B-CLL cells. Blood 113: 856-865, 2009.

54. Lupien M and Brown M: Cistromics of hormone-dependent cancer. Endocr Relat Cancer 16: 381-389, 2009.

55. Shaikhibrahim Z, Offermann A, Braun M, Menon R, Syring I, Nowak M, Halbach R, Vogel W, Ruiz C, Zellweger T, et al: MED12 overexpression is a frequent event in castration-resistant prostate cancer. Endocr Relat Cancer 21: 663-675, 2014.

56. Baca SC, Prandi D, Lawrence MS, Mosquera JM, Romanel A, Drier Y, Park K, Kitabayashi N, MacDonald TY, Ghandi M, et al: Punctuated evolution of prostate cancer genomes. Cell 153: 666-677, 2013

57. Barbieri CE, Baca SC, Lawrence MS, Demichelis F, Blattner M, Theurillat JP, White TA, Stojanov P, Van Allen E, Stransky N, et al: Exome sequencing identifies recurrent SPOP, FOXA1 and MED12 mutations in prostate cancer. Nat Genet 44: 685-689, 2012.

58. Grasso CS, Wu YM, Robinson DR, Cao X, Dhanasekaran SM, Khan AP, Quist MJ, Jing X, Lonigro RJ, Brenner JC, et al: The mutational landscape of lethal castration-resistant prostate cancer. Nature 487: 239-243, 2012

59. Risheg H, Graham JM Jr, Clark RD, Rogers RC, Opitz JM, Moeschler JB, Peiffer AP, May M, Joseph SM, Jones JR, et al: A recurrent mutation in MED12 leading to R961W causes Opitz-Kaveggia syndrome. Nat Genet 39: 451-453, 2007.

60. Schwartz CE, Tarpey PS, Lubs HA, Verloes A, May MM, Risheg H, Friez MJ, Futreal PA, Edkins S, Teague J, et al: The original Lujan syndrome family has a novel missense mutation (p.N1007S) in the MED12 gene. J Med Genet 44: 472-477, 2007.

61. Vulto-van Silfhout AT, de Vries BB, van Bon BW, Hoischen A, Ruiterkamp-Versteeg M, Gilissen C, Gao F, van Zwam M, Harteveld CL, van Essen AJ, et al: Mutations in MED12 cause X-linked Ohdo syndrome. Am J Hum Genet 92: 401-406, 2013.

62. Chen M, Carkner R and Buttyan R: The hedgehog/Gli signaling paradigm in prostate cancer. Expert Rev Endocrinol Metab 6: 453-467, 2011.

63. Zhou H, Kim S, Ishii S and Boyer TG: Mediator modulates Gli3-dependent Sonic hedgehog signaling. Mol Cell Biol 26: 8667-8682, 2006. 
64. Zhou H, Spaeth JM, Kim NH, Xu X, Friez MJ, Schwartz CE and Boyer TG: MED12 mutations link intellectual disability syndromes with dysregulated GLI3-dependent Sonic Hedgehog signaling. Proc Natl Acad Sci USA 109: 19763-19768, 2012.

65. Banerji S, Cibulskis K, Rangel-Escareno C, Brown KK, Carter SL, Frederick AM, Lawrence MS, Sivachenko AY, Sougnez C, Zou L, et al: Sequence analysis of mutations and translocations across breast cancer subtypes. Nature 486: 405-409, 2012.

66. Chao X, Tan W, Tsang JY, Tse GM, Hu J, Li P, Hou J, Li M, He J and Sun P: Clinicopathologic and genetic features of metaplastic breast cancer with osseous differentiation: A series of 6 cases. Breast Cancer 28: 1100-1111, 2021.

67. Shah SP, Roth A, Goya R, Oloumi A, Ha G, Zhao Y, Turashvili G, Ding J, Tse K, Haffari G, et al: The clonal and mutational evolution spectrum of primary triple-negative breast cancers. Nature 486: 395-399, 2012.

68. Zhang Y, Shi X, Zhang J, Chen X, Zhang P, Liu A and Zhu T: A comprehensive analysis of somatic alterations in Chinese ovarian cancer patients. Sci Rep 11: 387, 2021.

69. Zhang S, Liu F, Halfmann P, Behrens RT, Liu P, McIlwain SJ, Ong IM, Donahue K, Wang Y, Kawaoka Y, et al: Mediator complex subunit 12 is a gatekeeper of SARS-CoV-2 infection in breast cancer cells. Genes Dis: Aug 17, 2021. doi: 10.1016/j. gendis.2021.08.001 (Epub ahead of print).

70. Zhang S, O'Regan $\mathrm{R}$ and $\mathrm{Xu} \mathrm{W}$ : The emerging role of mediator complex subunit 12 in tumorigenesis and response to chemotherapeutics. Cancer 126: 939-948, 2020.

71. Wang L, Zeng H, Wang Q, Zhao Z, Boyer TG, Bian X and Xu W: MED12 methylation by CARM1 sensitizes human breast cancer cells to chemotherapy drugs. Sci Adv 1: e1500463, 2015.

72. Peng BL, Li WJ, Ding JC, He YH, Ran T, Xie BL, Wang ZR, Shen HF, Xiao RQ, Gao WW, et al: A hypermethylation strategy utilized by enhancer-bound CARM1 to promote estrogen receptor $\alpha$-dependent transcriptional activation and breast carcinogenesis. Theranostics 10: 3451-3473, 2020.

73. Schleicher EM, Dhoonmoon A, Jackson LM, Clements KE, Stump CL, Nicolae CM and Moldovan GL: Dual genome-wide CRISPR knockout and CRISPR activation screens identify mechanisms that regulate the resistance to multiple ATR inhibitors. PLoS Genet 16: e1009176, 2020.
74. Reaper PM, Griffiths MR, Long JM, Charrier JD, Maccormick S, Charlton PA, Golec JM and Pollard JR: Selective killing of ATM- or p53-deficient cancer cells through inhibition of ATR. Nat Chem Biol 7: 428-430, 2011.

75. Reinhardt HC, Aslanian AS, Lees JA and Yaffe MB: p53-deficient cells rely on ATM- and ATR-mediated checkpoint signaling through the p38MAPK/MK2 pathway for survival after DNA damage. Cancer Cell 11: 175-189, 2007.

76. Vendetti FP, Lau A, Schamus S, Conrads TP, O'Connor MJ and Bakkenist CJ: The orally active and bioavailable ATR kinase inhibitor AZD6738 potentiates the anti-tumor effects of cisplatin to resolve ATM-deficient non-small cell lung cancer in vivo. Oncotarget 6: 44289-44305, 2015.

77. Luo XL, Deng CC, Su XD, Wang F, Chen Z, Wu XP, Liang SB, Liu JH and Fu LW: Loss of MED12 induces tumor dormancy in human epithelial ovarian cancer via downregulation of EGFR. Cancer Res 78: 3532-3543, 2018.

78. Srivastava $\mathrm{S}$ and Kulshreshtha R: Insights into the regulatory role and clinical relevance of mediator subunit, MED12, in human diseases. J Cell Physiol 236: 3163-3177, 2021

79. Rosell R, Bivona TG and Karachaliou N: Genetics and biomarkers in personalisation of lung cancer treatment. Lancet 382: 720-731, 2013.

80. Broude EV, Győrffy B, Chumanevich AA, Chen M, McDermott MS, Shtutman M, Catroppo JF and Roninson IB: Expression of CDK8 and CDK8-interacting Genes as potential biomarkers in breast cancer. Curr Cancer Drug Targets 15: 739-749, 2015

81. Ma D, Chen X, Shen XB, Sheng LQ and Liu XH: Binding patterns and structure-activity relationship of CDK8 inhibitors. Bioorg Chem 96: 103624, 2020.

This work is licensed under a Creative Commons Attribution-NonCommercial-NoDerivatives 4.0 International (CC BY-NC-ND 4.0) License. 